\title{
Translationally Invariant States and the Spectrum Ideal in the Algebra of Test Functions for Quantum Fields
}

\author{
Jakob Yngvason \\ The Science Institute, University of Iceland, Reykjavik, Iceland
}

\begin{abstract}
A class of states on Borchers' tensor algebra is constructed. These states are invariant under the translation group and fulfill the spectrum condition. This leads to a characterization of the linear span of all such states in terms of a simple continuity property.
\end{abstract}

\section{Introduction}

In the algebraic formulation of Wightman's axioms, quantum fields correspond to a class of positive, linear functionals on a topological $*$-algebra, the tensor algebra over a space of test functions [1-3]. The conditions which distinguish Wightman functionals from other positive functionals on the algebra are invariance under a group of automorphisms and the requirement that the functionals vanish on two prescribed ideals. Various general aspects of the positive linear functionals on this algebra have been studied, e.g. in [4-14], cf. also [15] and literature quoted therein. The invariance condition and the two ideals play almost no role in these works, however. In the present paper, we want to take into account a part of the automorphism group, the translations of space-time, and one of the ideals, which corresponds to the spectrum condition for energy and momentum. We give a characterization of the linear span of all positive, invariant functionals satisfying a general spectrum condition. From this characterization follows in particular, that the invariant positive functionals span a dense subspace of the space of all invariant functionals and that the spectrum ideal is the intersection of the left kernels of the positive invariant functionals which annihilate it.

It is perhaps worthwhile to point out some differences between the present framework and the theory of $C^{*}$-algebras. On a $C^{*}$-algebra, there are just as many invariant states for a given group of automorphisms as there are invariant linear functionals. This is so because the Jordan decomposition of a linear functional automatically preserves invariance. Moreover, for an amenable group of automorphisms, one can construct an invariant functional from an arbitrary functional by using an invariant mean. For the tensor algebra these methods do not work. First, 
there is no minimal decomposition for linear functionals, and in fact, not every continuous functional is a linear combination of positive ones [7]. Secondly, an invariant mean is of almost no use, because the algebra is not a normed algebra, and the combination of the automorphisms with a non-invariant functional will in general lead to an unbounded function on the group. These matters are discussed a little further in [16]. In a $C^{*}$-algebra, every left ideal is the intersection of the left kernels of pure states and an analogous statement holds for the two-sided ideals. For the tensor algebra, this is far from being true. For a better mathematical understanding of the Wightman framework, it would be desirable to know which ideals and automorphisms go well together with the positive, linear functionals on the tensor algebra. The present paper may be considered as a part of such a programme. To a certain extent, this applies also to a joint paper of the author with Borchers [17], where the ideal in question is the one generated by all commutators. The main objective of that paper, however, was to find conditions on Schwinger functions, under which they have a representation by a measure on a space of distributions. If the requirement of Euclidean invariance for the measure is added, this problem also has a bearing on the present paper, and the last section is devoted to some remarks on this point. In particular, it contains a simple proof of the fact that, contrary to what is the case for Schwinger functions, the time ordered functions of field theory can never have a representation by a translationally invariant, complex measure on a space of distributions.

We now give a summary of the other parts of the paper. After some preparations in Sect. 2, we discuss in Sect. 3 continuous, translationally invariant seminorms on Schwartz space $\mathscr{S}$ and the tensor algebra $\mathscr{P}$. The collection of all such seminorms defines a topology on $\mathscr{S}$, in which $\mathscr{S}$ is no longer a nuclear space, and the subclass of invariant Hilbert seminorms defines a strictly weaker topology. It is this latter topology which is relevant for the invariant positive functionals and we give an explicit description of it. In Sect. 4, we show that every invariant Hilbert seminorm annhilating the spectrum ideal can be dominated by a positive linear functional with the same property. This result is analogous to Theorem 1 in [7] but the proof is more complicated and it turns out that the geometrical shape of the spectrum is important. A characterization of the linear span of all invariant states with spectrum condition and related results in Sect. 5 follow as simple corollaries.

\section{Notations and Preliminaries}

We use mostly the same notation as in [7]. The test function algebra $\mathscr{P}$ is the completed tensor algebra over Schwartz space $\mathscr{S}=\mathscr{S}_{1}=\mathscr{S}\left(\mathbb{R}^{d}\right)$. It is thus a direct sum

$$
\mathscr{S}=\bigoplus_{n=0}^{\infty} \mathscr{S}_{n}
$$

with $\mathscr{S}_{0}=\mathbb{C}$ and $\mathscr{S}_{n}=\mathscr{S}\left(\mathbb{R}^{n \cdot d}\right)$. Its elements are sequences $\underline{f}=\left(f_{0}, f_{1}, \ldots\right)$ with all but finitely many $f_{n} \in \mathscr{S}_{n}$ equal to 0 . Addition and multiplication by scalars are defined in an obvious way, and the product of two elements by

$$
(\underline{f} \times g)_{n}\left(x_{1}, \ldots, x_{n}\right)=\sum_{v+\mu=n} f_{v}\left(x_{1}, \ldots, x_{v}\right) g_{\mu}\left(x_{v+1}, \ldots, x_{n}\right) .
$$


The adjoint $\underline{f}^{*}$ of $\underline{f} \in \mathscr{S}$ is defined by

$$
\left(\underline{f}^{*}\right)_{n}\left(x_{1}, \ldots, x_{n}\right)=\overline{f_{n}\left(x_{n}, \ldots, x_{1}\right)},
$$

where the bar is complex conjugation. For Fourier transforms

$$
\tilde{f}_{n}\left(p_{1}, \ldots, p_{n}\right)=\frac{1}{(2 \pi)^{n d / 2}} \int e^{i \Sigma x_{k} p_{k}} f\left(x_{1}, \ldots, x_{n}\right) d x_{1} \ldots d x_{n},
$$

this means

$$
\widetilde{f_{n}^{*}}\left(p_{1}, \ldots, p_{n}\right)=\overline{\tilde{f}_{n}\left(-p_{n}, \ldots,-p_{1}\right)} \text {. }
$$

If $\mathscr{M}$ is a subset of $\mathscr{S}$, we denote by $\mathscr{M}^{(n)}$ its intersection with $\mathscr{S}_{n}$, i.e. $\mathscr{M}^{(n)}=\left\{f_{n} \in \mathscr{S}_{n} \mid\left(0, \ldots, f_{n}, 0, \ldots\right) \in \mathscr{M}\right\}$.

The topology on $\mathscr{S}$ is the locally convex direct sum of the topologies of the $\mathscr{S}_{n}$, and there are many equivalent descriptions of this topology in terms of seminorms (cf. e.g. [7]). We recall from [7] a notation for tensor products of seminorms, which we shall use in the sequel. If $\|\cdot\|^{\prime}$ is a continuous seminorm on $\mathscr{S}_{n}$ and $\|\cdot\|^{\prime \prime}$ on $\mathscr{S}_{m}$, then $\|\cdot\|^{\prime} \otimes_{\pi}\|\cdot\|^{\prime \prime}$ is a continuous seminorm on $\mathscr{S}_{n+m} \simeq \mathscr{S}_{n} \otimes \mathscr{S}_{m}$, defined as the largest seminorm $\|\cdot\|$ with the property $\|f \otimes g\|$ $=\|f\|^{\prime}\|g\|^{\prime \prime}$ for $f \in \mathscr{S}_{n}, g \in \mathscr{S}_{m}$. If $\|\cdot\|^{\prime},\|\cdot\|^{\prime \prime}$ are Hilbert seminorms, corresponding to scalar products $(\cdot, \cdot)^{\prime}$ and $(\cdot, \cdot)^{\prime \prime}$, then $\|\cdot\|^{\prime} \otimes_{\sigma}\|\cdot\|^{\prime \prime}$ is defined as the Hilbert seminorm on $\mathscr{S}_{n+m}$, corresponding to the scalar product

$$
(f \otimes g, h \otimes k)=(f, h)^{\prime}(g, k)^{\prime \prime} .
$$

In general, $\|\cdot\|^{\prime} \otimes_{\sigma}\|\cdot\|^{\prime \prime}$ is strictly weaker than $\|\cdot\|^{\prime} \otimes_{\pi}\|\cdot\|^{\prime \prime}$. Since $\mathscr{S}_{m}$ is a nuclear space, there is, however, always a continuous Hilbert seminorm $\|\cdot\|^{\prime \prime \prime}$ on $\mathscr{S}_{m}$ such that

$$
\|\cdot\|^{\prime} \otimes_{\pi}\|\cdot\|^{\prime \prime} \leqq\|\cdot\|^{\prime} \otimes_{\sigma}\|\cdot\|^{\prime \prime \prime}
$$

The continuous linear functionals on $\mathscr{S}$ have the form $T=\left(T_{0}, T_{1}, \ldots\right)$ with $T_{n} \in \mathscr{S}_{n}^{\prime}$ and $T(\underline{f})=\Sigma T_{n}\left(f_{n}\right)$. A functional $T$ is called hermitan if $T\left(\underline{f}^{*}\right)=\overline{T(f)}$, and positive if $T\left(\underline{f}^{*} \times \underline{f}\right) \geqq 0$ for all $\underline{f}$. The space of all continuous, linear functionals on $\mathscr{S}$ is denoted by $\mathscr{L}^{\prime}$, its hermitan part by $\mathscr{S}_{h}^{\prime}$, and the cone of positive linear functionals by $\mathscr{S}^{+\prime}$. For $T \in \mathscr{Q}^{+\prime}$ we have $T_{0}>0$ unless $T \equiv 0$. If $T \in \mathscr{S}^{+\prime}$ is normalized so that $T_{0}=1$, we call $T$ a state. When we have occasion to talk about a topology on $\mathscr{S}_{n}^{\prime}$ and $\mathscr{S}^{\prime}$, this is understood to be the strong topology.

The translation group $\mathbb{R}^{d}$ operates on $\mathscr{S}$ as a group of automorphisms: For $a \in \mathbb{R}^{d}$ and $\underline{f} \in \mathscr{\mathscr { S }}$ we define

$$
\left(\alpha_{a} \underline{f}\right)_{n}\left(x_{1}, \ldots, x_{n}\right)=f_{n}\left(x_{1}-a, \ldots, x_{n}-a\right) .
$$

In terms of the Fourier transform this is

$$
\left(\widetilde{\alpha_{a} f}\right)_{n}\left(p_{1}, \ldots, p_{n}\right)=e^{i\left(p_{1}+\ldots+p_{n}\right) \cdot a} \tilde{f}\left(p_{1}, \ldots, p_{n}\right) .
$$

Call $\mathscr{S}_{\text {inv }}^{\prime}$ the subspace of all continuous, linear functionals which are invariant under these transformations.

We omit the straightforward proof of the next proposition. 
2.1. Proposition. The following *-invariant, closed subspaces of $\mathscr{P}$ are all equal:

(i) Closed linear span $\left\{\underline{f}-\alpha_{a} f \underline{f} \in \underline{\mathscr{S}}, a \in \mathbb{R}^{d}\right\}$.

(ii) Closure of $\left\{\int \alpha_{a} f h(a) d a \mid \underline{f} \in \underline{\mathscr{P}}, h \in \mathscr{S}_{1}, \tilde{h}(0)=0\right\}$.

(iii) $\left\{\underline{f} \mid f_{0}=0\right.$ and $\int f_{n}\left(x_{1}+a, \ldots, x_{n}+a\right) d a \equiv 0$ for all $\left.n\right\}$.

(iv) $\left\{\underline{f} \mid f_{0}=0, \tilde{f}_{n}\left(p_{1}, \ldots, p_{n}\right)=0\right.$ if $\left.p_{1}+\ldots+p_{n}=0\right\}$.

(v) $\bigcap_{T \in \mathscr{S}_{\text {inv }}^{\prime}} \operatorname{ker} T$.

2.2. Notation. The subspace described in Proposition 2.1 is denoted $\mathscr{M}_{\text {inv }}$.

The dual group of $\mathbb{R}^{d}$ can be identified with $\mathbb{R}^{d}$ itself as usual, by means of the Minkowski scalar product

$$
x \cdot y=x^{0} y^{0}-x^{1} y^{1}-\ldots-x^{d-1} y^{d-1} .
$$

2.3. Definition. Let $S \subset \mathbb{R}^{d}$ be a closed set with $0 \in S$. The spectrum ideal $\mathscr{L}_{S}$ corresponding to $S$ is the closed left ideal in $\mathscr{S}$ generated by elements of the form

$$
\int \alpha_{a} f h(a) d a
$$

with $\underline{f} \in \mathscr{\mathscr { S }}$ and $h \in \mathscr{S}_{1}$, such that the Fourier transform $\tilde{h}$ vanishes on $S$.

A translationally invariant state $T$ on $\mathscr{S}$ gives rise via GNS construction to a continuous unitary representation of the translation group, and the joint spectrum of the generators of this representation is contained in $S$ if and only if $T$ vanishes on $\mathscr{L}_{S}$. The case of interest in quantum field theory is of course $S=V^{+}$(forward light cone) or some Lorentz invariant subset of it containing 0 .

Every hermitian functional which annihilates $\mathscr{L}_{S}$ vanishes also on the right ideal

$$
\mathscr{L}_{S}^{*}=\left\{\underline{f} \mid \underline{f}^{*} \in \mathscr{L}_{S}\right\} .
$$

Moreover, the spectrum ideal is mainly of interest in connection with invariant functionals. With this in mind, we introduce

2.4. Notation. $\mathscr{M}_{S}:=$ closure of $\left(\mathscr{L}_{S}+\mathscr{L}_{S}^{*}\right)$

$$
\hat{\mathscr{M}}_{S}:=\text { closure of }\left(\mathscr{L}_{S}+\mathscr{M}_{\text {inv }}\right) \text {. }
$$

We shall soon see that $\hat{\mathscr{M}}_{S}$ is automatically *-invariant, so $\mathscr{M}_{S} \subset \hat{\mathscr{M}}_{S}$. From the bipolar theorem we have

\subsection{Proposition.}

$$
\begin{aligned}
& \mathscr{M}_{S}=\bigcap_{T \in \mathscr{\mathscr { S }}_{h}^{\prime} \cap \mathscr{L}^{\perp}} \operatorname{ker} T \\
& \hat{\mathscr{M}}_{\mathrm{S}}=\bigcap_{T \in \mathscr{S}_{\text {inv }} \cap \mathscr{L}_{S}^{\perp}} \operatorname{ker} T .
\end{aligned}
$$

Here $\perp$ stands for the orthogonal complement in the dual space. Note that all these spaces are the direct sum of their projections onto $\mathscr{S}_{n}$. In order to describe these and related spaces explicitly, it is convenient to make a transformation of coordinates on $\mathbb{R}^{n \cdot d}$ and define

$$
q_{k}=\sum_{v=n+1-k}^{n} p_{v} \quad k=1, \ldots, n, \quad p_{v} \in \mathbb{R}^{d}
$$


We write these coordinates in inverse order, thus : $\left(q_{n}, q_{n-1}, \ldots, q_{1}\right)$. (This somewhat strange convention turns out to be convenient in the proof of Theorem 4.5.) The Fourier transform of a function $f \in \mathscr{S}_{n}$ expressed in these coordinates will simply be written $f\left(q_{n}, \ldots, q_{1}\right)$. The *-operation is, in this notation,

$$
f^{*}\left(q_{n}, \ldots, q_{1}\right)=\overline{f\left(-q_{n}, q_{1}-q_{n}, \ldots, q_{n-1}-q_{n}\right)} .
$$

2.6. Proposition. (i) $\mathscr{L}_{\gamma}=\left\{\underline{f} \mid f_{0}=0, f_{n}\left(q_{n}, \ldots, q_{1}\right)=0\right.$ on $\left.S \times \ldots \times S\right\}, \mathscr{L}_{S}^{*}=\left\{\underline{f} \mid f_{0}=0\right.$, $f_{n}\left(q_{n}, \ldots, q_{1}\right)=0$ if $q_{n} \in-S$ and $q_{v} \in S+q_{n}$ for $\left.v=1, \ldots, n-1\right\}$.

(ii) $\hat{\mathscr{M}}_{S}=\left\{\underline{f} \mid f_{0}=0, f_{n}\left(q_{n}, \ldots, q_{1}\right)=0\right.$ on $\left.\{0\} \times S \times \ldots \times S\right\}$.

(iii) $\mathscr{M}_{S}$ is ${ }^{*}$-invariant, and $\mathscr{M}_{S} \subset \mathscr{M}_{S}$. If $S \cap-S=\{0\}$ and 0 is an isolated point of $S$, then $\mathscr{M}_{\mathrm{S}}=\hat{\mathscr{M}}_{\mathrm{S}}$.

(iv) There is a natural isomorphism

$$
\mathscr{S} / \mathscr{L}_{S} \simeq \bigoplus_{n=0}^{\infty}\left(\mathscr{S}_{1} / \mathscr{L}_{S}^{(1)}\right)^{\hat{\otimes} n}
$$

Here $\hat{\otimes} n$ denotes the completed $n$-fold tensor product with respect to the q-variables.

The proof is straightforward and is given in the appendix.

2.7. Remarks. (i) If 0 is not an isolated point of $S$ (e.g. if $S$ is the whole forward light cone), then the functions in $\mathscr{M}_{S}^{(n)}$ will in general vanish with all derivatives for $q_{n}=0$, so $\mathscr{M}_{S}$ is a proper subset of $\hat{\mathscr{M}}_{S}$.

(ii) For sufficiently regular $S$, one can show (using Whitney's extension theorem) that $\mathscr{L}_{S}+\mathscr{L}_{S}^{*}$ and $\mathscr{L}_{S}+\mathscr{M}_{\text {inv }}$ are already closed.

\section{Translationally Invariant Seminorms}

In this section we first describe a basis for the set of all continuous seminorms on $\mathscr{S}_{1}$, which are invariant under the translation group $\mathbb{R}^{d}$. Next we define some strictly weaker topologies with the aid of $L_{p}$-norms of Fourier transforms. By introducing difference variables, one can extend these topologies to $\mathscr{S}_{n}$ and $\mathscr{S}$, and the topology generated by all continuous translationally invariant Hilbert seminorms on $\mathscr{\mathscr { S }}$ vanishing on $\mathscr{L}_{\mathrm{S}}$ is shown to be identical to one of them.

3.1. Proposition. Let $\|\cdot\|$ be a continuous seminorm on $\mathscr{S}_{1}$, such that $a \mapsto\left\|\alpha_{a} f\right\|$ is a bounded function of $a \in \mathbb{R}^{d}$ for all $f \in \mathscr{S}_{1}$. Then $\|\cdot\|$ is dominated by one of the invariant seminorms

$$
\|f\|_{1, N}:=\sum_{|v|=0}^{N} \int\left|D^{v} f(x)\right| d x .
$$

Proof. If $a \mapsto\left\|\alpha_{a} f\right\|$ is bounded for all $f$, we infer from the Banach-Steinhaus theorem that $\left\|\alpha_{a} f\right\| \leqq\|f\|^{\prime}$ for all $a$ and $f$ with some continuous seminorm $\|\cdot\|^{\prime}$ on $\mathscr{S}_{1}$. Divide $\mathbb{R}^{d}$ into cubes $Q_{k}$ with side lengths 1 , indexed by corner points $k \in \mathbb{Z}^{d}$, such that $Q_{k}=Q_{0}+k$. Choose a $C^{\infty}$-function $\chi_{0}$ with supp $\chi_{0} \subset \bigcup_{|l| \leqq 1} Q_{l}=: \hat{Q}_{0}$ and $\sum_{k} \chi_{k} \equiv 1$, where $\chi_{k}:=\alpha_{k} \chi_{0}$. We have $\|f\| \leqq\left\|\sum_{k} f \chi_{k}\right\| \leqq \sum_{k}\left\|f \chi_{k}\right\| \leqq \sum_{k}\left\|\left(\alpha_{k} f\right) \chi_{0}\right\|^{\prime}$. 
Since $\|\cdot\|^{\prime}$ is continuous, there is an $N$ such that

$$
\left\|\left(\alpha_{k} f\right) \chi_{0}\right\|^{\prime} \leqq \text { const } \sum_{|v|=0}^{N} \int_{\hat{Q}_{0}}\left|D^{v}\left\{\left(\alpha_{k} f\right) \chi_{0}\right\}\right| d x \leqq \text { const } \sum_{|v|=0}^{N} \int_{\hat{Q}_{0}}\left|D^{v} f(x-k)\right| d x .
$$

Thus,

$$
\begin{aligned}
\|f\| & \leqq \text { const } \sum_{|v|=0}^{N} \sum_{k} \int_{\hat{Q}_{0}}\left|D^{v} f(x-k)\right| d x \\
& \leqq \text { const } \sum_{|v|=0}^{N} \sum_{k} \int_{Q_{k}}\left|D^{v} f(x)\right| d x \leqq \text { const } \sum_{|v|=0}^{N} \int\left|D^{v} f(x)\right| d x .
\end{aligned}
$$

The invariant seminorms $\|\cdot\|_{1, N}$ thus form a basis among all continuous, invariant seminorms on $\mathscr{S}_{1}$. Call $\mathfrak{I}_{\text {inv }}$ the topology which they define on $\mathscr{S}_{1}$. The dual space of $\mathscr{S}_{1}$ in this topology consists precisely of the bounded distributions, cf. [18], p. 200.

Let $\varrho$ be a tempered, positive measure on $\mathbb{R}^{d}$ and $1 \leqq r<\infty$. Define for $f \in \mathscr{S}_{1}$

If $N \in \mathbb{N}$, put

$$
\|f\|_{r, \varrho}^{\sim}:=\left(\int|\tilde{f}(q)|^{r} d \varrho(q)\right)^{1 / r} .
$$

$$
\|f\|_{\infty, N}^{\sim}:=\sup _{q}\left|\left(1+|q|^{N}\right) \tilde{f}(q)\right| .
$$

For fixed $r, 1 \leqq r \leqq \infty$, call $\tilde{\mathfrak{I}}_{r}$ the topology defined by all $\|\cdot\|_{r, \varrho}^{\sim}$ respectively $\|\cdot\|_{\infty, N}^{\sim}$. More generally, for an arbitrary closed set $S$, define $\tilde{\mathfrak{I}}_{r, S}$ by considering only $\varrho^{\prime} \mathrm{s}$ with support in $S$, respectively (for $r=\infty$ ) by the seminorms

$$
\|f\|_{\infty, N, S}^{\sim}:=\sup _{q \in S}\left|\left(1+|q|^{N}\right) \tilde{f}(q)\right| .
$$

3.2. Proposition. (i) If $1 \leqq r<s \leqq \infty$, then $\tilde{\mathfrak{T}}_{r, S}$ is weaker than $\tilde{\mathfrak{T}}_{s, S}$; for general $S$ strictly weaker. Moreover, $\tilde{\mathfrak{I}}_{\infty}$ is strictly weaker than $\mathfrak{I}_{\text {inv }}$.

(ii) The continuous linear functionals on $\mathscr{S}$ in the topologies $\tilde{\mathfrak{T}}_{r, s}$ are the same for all $r$ and have the form

$$
T(f)=\int \tilde{f}(q) d \mu(q),
$$

where $\mu$ is a tempered, complex measure on $\mathbb{R}^{d}$ with support in $S$. On the other hand, the dual space of $\mathscr{S}\left[\mathfrak{I}_{\text {inv }}\right]$ is strictly larger than that of $\mathscr{S}\left[\tilde{\mathfrak{I}}_{\infty}\right]$.

Proof. (i) If $\varrho$ is a positive measure with support in $S$ and $\left(1+|q|^{N}\right)^{-1}$ is $\varrho$ integrable, then $\|\cdot\|_{r, \varrho} \leqq$ const $\|\cdot\|_{\infty, N, S}$, and if $r<s<\infty$, then $\|\cdot\|_{r, \varrho} \leqq\|\cdot\|_{s, \varrho^{\prime}}$ with $\varrho^{\prime}=\operatorname{const}\left(1+|q|^{N}\right) \varrho$, by Hölder's inequality. It is a simple exercise in measure theory to show that $\tilde{\mathfrak{T}}_{r, S}$ is strictly weaker than $\tilde{\mathfrak{T}}_{s, S}$ (unless $S$ contains only a countable number of points). That $\tilde{\mathfrak{I}}_{\infty}$ is strictly weaker than $\mathfrak{I}_{\text {inv }}$ follows from (ii).

(ii) If $T \in \mathscr{S}^{\prime}$ satisfies $|T(f)| \leqq$ const $\|f\|_{\infty, N, S}$, it follows that $\left(1+|q|^{N}\right)^{-1} \tilde{T}(q)$ is continuous w.r.t. $\|\cdot\|_{\infty, 0, s}$ and thus a bounded complex measure $\mu_{0}$. Hence $T$ is represented by the tempered measure $\left(1+|q|^{N}\right) \mu_{0}$. Conversely, if $T$ has such a representation, it is $\tilde{\mathfrak{I}}_{r, s}$-continuous for all $r$. On the other hand, there exist bounded (and thus $\mathfrak{I}_{\text {inv }}$-continuous) distributions which are not Fourier transforms of complex measures. (Example: Heaviside step function on $\mathbb{R}$, whose Fourier transform is principal value $1 / q$.) Thus $\mathfrak{I}_{\text {inv }} \neq \tilde{\mathfrak{T}}_{\infty}$. 
Until now we have only considered invariant seminorms on $\mathscr{S}_{1}$. In order to carry this over to $\mathscr{S}_{n}$, we introduce the coordinates $\xi_{1}=x_{1}, \xi_{k}=x_{k}-x_{k-1}$, $k=2, \ldots, n$ on $\mathbb{R}^{n d}$. In Fourier space this corresponds to replacing $\left(p_{1}, \ldots, p_{n}\right)$ by $\left(q_{n}, \ldots, q_{1}\right)$ as in the preceding section. (In our convention, $q_{k}$ is the conjugate momentum to $\xi_{n+1-k}$.) The translation group operates only on $\xi_{1}$ (respectively $q_{n}$ ), and we can write $\mathscr{S}_{n}=\mathscr{S}_{1} \hat{\otimes} \mathscr{S}_{n-1}$, where the functions in the first factor depend only on $\xi_{1}$ (respectively $q_{n}$ ) and in the second factor on $\xi_{2}, \ldots, \xi_{n}$ (respectively $q_{n-1}, \ldots, q_{1}$ ). In the remainder of this section, $\otimes$ stands for this decomposition of $\mathscr{S}_{n}$.

3.3. Definition. The topology $\mathfrak{I}_{\text {inv }}$ on $\mathscr{\mathscr { S }}$ is defined by all seminorms of the form

$$
\|\underline{f}\|=\sum_{n=0}^{\infty}\left\|f_{n}\right\|_{(n)}
$$

with $\|\cdot\|_{(n)}=\|\cdot\|_{1, N_{n}} \otimes_{\pi}\|\cdot\|_{(n-1)}^{\prime}$, where $N_{n} \in \mathbb{N}$ and $\|\cdot\|_{(n-1)}^{\prime}$ is a continuous seminorm on $\mathscr{S}_{n-1}$.

In an analogous way we define $\tilde{\mathfrak{T}}_{r, S}$ on $\mathscr{\mathscr { S }}$, replacing $\|\cdot\|_{1, N_{n}}$ by $\tilde{\mathfrak{T}}_{r, S}$-continuous seminorms on $\mathscr{S}_{1}$, and requiring that the $\|\cdot\|_{(n-1)}^{\prime}$ vanish on $\mathscr{L}_{S}^{(n-1)}$.

3.4. Remark. Since $\mathscr{S}_{n-1}$ respectively $\mathscr{S}_{n-1} / \mathscr{L}_{S}^{(n-1)}$ are nuclear spaces, we can just as well use any other tensor product of seminorms which lies between $\otimes_{\pi}$ and $\otimes_{\varepsilon}$. Also, it is immaterial whether we define $\|\cdot\|$ from the $\|\cdot\|_{(n)}$ as above, or if $\|\underline{f}\|:=\sup _{n}\left\|f_{n}\right\|_{(n)}$, or $\|\underline{f}\|:=\left(\sum_{n}\left\|f_{n}\right\|_{(n)}^{p}\right)^{1 / p}$ with $1 \leqq p<\infty$. In particular, the topology $\tilde{\mathfrak{I}}_{2, S}^{n}$ can be defined by Hilbert seminorms of the type

$$
\|\underline{f}\|=\left(\sum_{n=0}^{\infty}\left\|f_{n}\right\|_{(n)}^{2}\right)^{1 / 2},
$$

with $\|\cdot\|_{(n)}=\|\cdot\|_{2, \varrho_{n}} \otimes_{\sigma}\|\cdot\|_{(n-1)}^{\prime}$, where the $\varrho_{n}$ are positive, tempered measures with support in $S$, and the $\|\cdot\|_{(n-1)}^{\prime}$ continuous Hilbert seminorms vanishing on $\mathscr{L}_{S}^{(n-1)}$.

3.5. Proposition. The topology $\mathfrak{I}_{\text {inv }}$ on $\mathscr{S}$ is equal to the topology defined by all continuous seminorms on $\mathscr{S}$ such that $a \mapsto\left\|\alpha_{a} \underline{f}\right\|$ is a bounded function for all $\underline{f} \in \underline{\mathscr{S}}$.

Proof. This is a combination of 3.1 with the Banach-Steinhaus theorem. From 3.1 follows that $f_{1} \mapsto\left\|f_{1} \otimes g_{n-1}\right\|$ is a $\mathfrak{I}_{\text {inv }}$-continuous seminorm on $\mathscr{S}_{1}$ for $g_{n-1} \in \mathscr{S}_{n-1}$ fixed. Now $\mathscr{S}_{1}$ is metrizable for $\mathfrak{I}_{\text {inv }}$, and $\mathscr{S}_{n-1}$ with the usual topology is a Fréchet space. By Theorem 3.4.1 in [19], $\left\|f_{1} \otimes g_{n-1}\right\|$ is therefore jointly continuous in $f_{1}$ and $g_{n-1}$, from which the assertion follows.

Since $\tilde{\mathfrak{T}}_{\infty, S}$ is a metrizable topology on $\mathscr{S}_{1}$, one can in a similar way show that $\tilde{\mathfrak{I}}_{\infty, S}$ on $\mathscr{S}$ is the topology generated by all continuous seminorms $\|\cdot\|$ vanishing on $\mathscr{L}_{S}$, such that $\| \int \alpha_{a} \underline{f}\left(h(a) d a \| \leqq \sup _{q \in S}|\tilde{h}(q)| \operatorname{const}(\underline{f})\right.$ for $\underline{f} \in \mathscr{\mathscr { S }}$ fixed and $h \in \mathscr{S}_{1}$. We shall not use that, but rather a slightly different statement, which is contained in the next proposition.

3.6. Proposition. Let $B$ be a translationally invariant sesquilinear form on $\mathscr{L}$, vanishing on $\mathscr{L}_{S}$. The following statements are equivalent: 
(i) For all $r, s \geqq 1$, with $1 / r+1 / s=1$, there is a $\tilde{\mathfrak{T}}_{r, s^{-}}$continuous seminorm $\|\cdot\|_{r}$ and a $\tilde{\mathfrak{I}}_{s, s^{-}}$continuous seminorm $\|\cdot\|_{s}$, such that

$$
|B(\underline{f}, \underline{g})| \leqq\|\underline{f}\|_{\boldsymbol{r}}\|\underline{g}\|_{\mathrm{s}} \text { for all } \underline{f}, \underline{g} .
$$

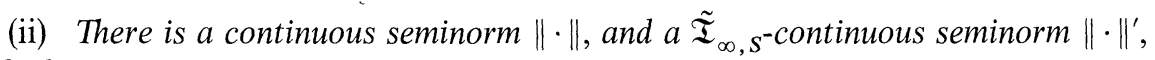
such that

$$
|B(\underline{f}, \underline{g})| \leqq\|\underline{f}\|\|\underline{g}\|^{\prime} \quad \text { for all } \underline{f}, \underline{g} .
$$

(iii) There is a continuous seminorm $\|\cdot\|$, such that

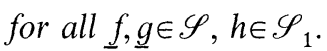

$$
\left|\int B\left(\underline{f}, \alpha_{a} g\right) h(a) d a\right| \leqq\|\underline{f}\|\|\underline{g}\| \sup _{q \in S}|\tilde{h}(q)|
$$

Proof. It is clear that (i) $\Rightarrow\left(\right.$ ii), and for a $\tilde{\mathfrak{I}}_{\infty . S}$-continuous seminorm as in Definition 3.3 it is also evident that $\left\|\int \alpha_{a} g h(a) d a\right\|^{\prime} \leqq \sup _{q \in S}|\tilde{h}(q)|\|\underline{g}\|^{\prime}$, so (ii) $\Rightarrow$ (iii). We now show (iii) $\Rightarrow$ (i). Let us write $h * g$ for $\int \alpha_{a} g h(a) d a$. Consider the mapping $(f, g, h) \mapsto B(f, h * g)$. It is antilinear in the first variable and linear in the others. Moreover, it is jointly continuous in all three variables, where $\mathscr{S}_{1}$ is equipped with the seminorm $\sup _{q \in S}|\tilde{h}(q)|$. By the abstract nuclear theorem (cf. [20], 7.4.3), there are equicontinuous sequences $\left\{L_{i}\right\}$ and $\left\{M_{i}\right\}$ in $\mathscr{L}_{S}^{\perp}$, a uniformly bounded sequence $\left\{v_{i}\right\}$ of complex measures with support in $S$ and a sequence $\left\{\lambda_{i}\right\}$ of positive numbers with $\sum \lambda_{i}<\infty$, such that

$$
B(\underline{f}, h * \underline{g})=\sum \lambda_{i} \overline{L_{i}(\underline{f})} v_{i}(\tilde{h}) M_{i}(\underline{g}) .
$$

We define a bounded, positive measure $v$ on $S$ by $v=\sum \lambda_{i}\left|v_{i}\right|$ and use the translation invariance of $B$ and equicontinuity of $\left\{L_{i}\right\}$ and $\left\{M_{i}\right\}$ to get

$$
\left|B\left(h_{1} * \underline{f}, h_{2} * \underline{g}\right)\right| \leqq \int\left|\tilde{h}_{1}(q) \tilde{h}_{2}(q)\right| d v(q) \cdot\|\underline{f}\|^{\prime}\|\underline{g}\|^{\prime},
$$

with some continuous seminorm $\|\cdot\|^{\prime}$, vanishing on $\mathscr{L}_{S}$. Consider the restriction of this seminorm to $\mathscr{S}_{n}$. For $g \in \mathscr{S}_{1}$ write $\tilde{g}\left(q_{n}\right)=\tilde{h}\left(q_{n}\right) \Phi_{N}\left(q_{n}\right)$ with $\Phi_{N}(q)=\left(1+|q|^{N}\right)^{-1}$. For a suitable $N=N_{n}, \Phi_{N_{n}} \otimes g_{(n-1)}$ belongs to the completion of $\mathscr{S}_{n}$ in $\|\cdot\|^{\prime}$ for all $g_{(n-1)} \in \mathscr{S}_{n-1}$, and $\left\|\Phi_{N_{n}} \otimes g_{(n-1)}\right\|^{\prime} \leqq\left\|g_{(n-1)}\right\|_{(n-1)}^{\prime \prime}$ with a continuous seminorm $\|\cdot\|_{(n-1)}^{\prime \prime}$ on $\mathscr{S}_{n-1}$, vanishing on $\mathscr{L}_{S}^{(n-1)}$. Hence, for $f, g \in \mathscr{S}_{1}$ and $f_{(m-1)} \in \mathscr{S}_{m-1}$, $g_{(n-1)} \in \mathscr{S}_{n-1}$, we have

$$
\begin{gathered}
\mid B\left(f \otimes f_{(m-1)}, g \otimes g_{(n-1)} \mid \leqq\right. \\
\int|\tilde{f}(q) \| \tilde{g}(q)|\left(1+|q|^{N_{m}}\right)\left(1+|q|^{N_{n}}\right) d v(q) \\
\cdot\left\|f_{(m-1)}\right\|^{\prime \prime}\left\|g_{(n-1)}\right\|^{\prime \prime},
\end{gathered}
$$

and (i) follows by Hölder's inequality.

As a corollary we obtain

3.7. Proposition. The topology $\tilde{\mathfrak{I}}_{2, S}$ on $\mathscr{\mathscr { S }}$ is equal to the topology generated by all translationally invariant, continuous Hilbert seminorms on $\mathscr{\mathscr { S }}$ which vanish on $\mathscr{L}_{S}$ 
Proof. By Remark 3.4, $\tilde{\mathfrak{I}}_{2, S}$ can be defined by invariant Hilbert seminorms. Conversely, suppose $(\cdot, \cdot)$ is a translationally invariant, continuous scalar product, vanishing on $\mathscr{L}_{s}$. The function $K\left(a-a^{\prime}\right)=\left(\alpha_{a^{\prime}} \underline{g}, \alpha_{a} \underline{g}\right)$ is the Fourier transform of a bounded, positive measure with total weight $K(0)=(g, g)$ by Bochner's theorem. It follows that the scalar product satisfies (iii) and hence (i) in Proposition 3.6.

\section{Construction of a Class of Invariant States}

Every translationally invariant, positive functional $T \in \mathscr{L}_{S}^{\perp}$ defines a continuous invariant Hilbert seminorm $\underline{f} \mapsto T\left(\underline{f}^{*} \times \underline{f}\right)^{1 / 2}$ vanishing on $\mathscr{L}_{S}$. We now want to show that conversely, any Hilbert seminorm with this property can be dominated by an invariant, positive functional in $\mathscr{L}_{S}^{\perp}$. This is not true for arbitrary $S$, however. It turns out that the following condition on the set $S$ is essential.

4.1. Definition. We say that a closed set $S \subset \mathbb{R}^{d}$ satisfies condition $(R)$, if there is a basis of continuous Hilbert scalar products $(\cdot, \cdot)_{k}, k=0,1,2, \ldots$ for the topology of $\mathscr{S}_{1} / \mathscr{L}_{S}^{(1)}$ such that

(1) $(\cdot, \cdot)_{0}$ is translationally invariant.

(2) For every $k$, there is a $k^{\prime}$ such that $\left|(f, g)_{k}\right|^{2} \leqq(f, f)_{0}(g, g)_{k^{\prime}}$ for all $f, g$.

4.2. Remark. Part (2) of the condition appears in the mathematical literature in a different context: it characterizes precisely those nuclear Fréchet spaces, which are isomorphic to closed subspaces of $\mathscr{S}$ [21].

Condition $(R)$ is a regularity condition on the set $S$. We now want to show that it is fulfilled in most cases of interest. An open connected set in $\mathbb{R}^{d}$ is usually called Lipschitzian, if its boundary is given locally by a function satisfying a Lipschitz condition (of order 1). This is a local condition on the set, invariant under diffeomorphisms. In order to exclude possible irregularities at infinity, let us agree here to use the term in a slightly stronger sense, and to mean that the image of the set under the map $q \mapsto q^{\prime}=q\left(1+|q|^{2}\right)^{-1 / 2}$ is also Lipschitzian at points lying on the boundary of the unit sphere.

4.3. Proposition. Suppose $S$ is a finite union of disjoint sets in $\mathbb{R}^{d}$, which are either the closure of open, Lipschitzian sets, or the closure of open, Lipschitzian subsets of lower dimensional submanifolds of $\mathbb{R}^{d}$. Furthermore, suppose the minimal distance between the connected components of $S$, within a sphere of radius $r$ around the origin, decreases at most like an inverse power of $r$, when $r \rightarrow \infty$. Then $S$ satisfies condition $(R)$.

Proof. Assume first that $S$ is the closure of an open, Lipschitzian set in $\mathbb{R}^{d}$. It follows from the extension theorems of Whitney, cf. e.g. [22], Theorem 3.1, p. 77, and Proposition 3.11, p. 79, that $\mathscr{S}_{1} / \mathscr{L}_{S}^{(1)}$ is isomorphic to the space $\mathscr{S}(S)$ of $C^{\infty}$ functions on $S$ of rapid decrease with the seminorms $\max \sup _{q \in S}\left|\left(1+|q|^{N}\right) D^{v} f(q)\right|$. $|v| \leqq N \quad q \in S$

The isomorphism is given by restricting functions in $\mathscr{S}$ to $S$. By a transformation of variables, $q \mapsto q^{\prime}=q\left(1+|q|^{2}\right)^{-1 / 2}$, we map $S$ onto a Lipschitzian set $S^{\prime}$, contained in the unit ball in $\mathbb{R}^{d}$, and $\mathscr{S}(S)$ onto a closed subspace of the space $\mathscr{D}\left(S^{\prime}\right)$ of $C^{\infty}$ - 
functions on $S^{\prime}$ with the seminorms max sup $\left|D^{v} f\left(q^{\prime}\right)\right|$. By a theorem of Zerner $|v| \leqq N \quad q^{\prime} \in S^{\prime}$

[23], c.f. also [24], Theorem 4, the expansion w.r.t. the orthogonal polynomials in $L_{2}\left(S^{\prime}, d q^{\prime}\right)$ defines an isomorphism between $\mathscr{D}\left(S^{\prime}\right)$ and the space o of rapidly decreasing sequences of complex numbers $\left(a_{v}\right)_{v=0,1} \ldots$ with its usual topology. This space obviously satisfies (2) with $\left(\left(a_{v}\right),\left(b_{v}\right)\right)_{k}=\sum_{v}(1+v)^{k} \bar{a}_{v} b_{v}$, and the same holds for all subspaces. In terms of functions of the original variable $q$, we have

$$
(f, g)_{0}=\int_{S} \overline{f(q)} g(q)\left(1+|q|^{2}\right)^{-1-d / 2} d q
$$

which is translationally invariant, so (1) is fulfilled.

If $S$ lies in a submanifold, diffeomorphic to $\mathbb{R}^{k}, k<d$, we note that all derivatives in directions normal to the manifold are suppressed in the quotient space $\mathscr{S}_{1} / \mathscr{L}_{S}^{(1)}$. One can therfore apply the preceding argument with $\mathbb{R}^{d}$ replaced by $\mathbb{R}^{k}$.

It is easy to extend this to arbitrary submanifolds by covering them with pieces diffeomorphic to $\mathbb{R}^{k}$, but this is a rather irrelevant generalization and we refrain from writing down a formal proof. Finally, if $S=\bigcup_{i=1}^{n} S_{i}$ with disjoint $S_{i}$ as above, we have $\mathscr{S}_{1} / \mathscr{L}_{S}^{(1)}=\bigoplus_{i=1}^{n}\left(\mathscr{S}_{1} / \mathscr{L}_{S_{i}}^{(1)}\right)$, because the last hypothesis of the proposition implies that one can split $f \in \mathscr{S}$ in a continuous way into a sum of functions $f_{i}$ with $f_{i}=f$ on $S_{i}$ and $f_{i}=0$ on $S_{j}, i \neq j$.

4.4. Remark. By the last proposition, condition $(R)$ is in particular fulfilled if $S$ is the forward light cone, or a Lorentz invariant subset of it, consisting of $\{0\}$, a finite number of mass shells and a continuum, as usual in relativistic quantum field theory.

Examples of closed sets, which do not satisfy condition $(R)$ may be constructed, either by considering sets with sharp "cusps," or by taking the union of an infinite number of disjoint sets. For instance, it can be shown that condition $(R)$ is violated for $S$ as above, but with an infinite number of mass shells below a continuum.

4.5. Theorem. Suppose $S$ satisfies condition $(R)$. Then for every continuous, translationally invariant Hilbert seminorm $\|\cdot\|$ vanishing on $\mathscr{L}_{S}$, there is a continuous, translationally invariant, positive linear functional $T$ in $\mathscr{L}_{S}^{\perp}$, such that

$$
\|\underline{f}\| \leqq T\left(\underline{f}^{*} \times f\right)^{1 / 2} \text { for all } \underline{f} \in \underline{\mathscr{S}} .
$$

Condition $(R)$ is also necessary for this to hold.

Proof. The components $T_{n} \in \mathscr{S}_{n}^{\prime}$ of $T$ will be constructed by induction over $n$ as in Theorem 1 in [7]. The odd components can be taken to be 0 , and the even components $T_{2 m}$ are defined by means of suitable scalar products on $\mathscr{S}_{m}$. At each step, one must have two estimates for $T_{2 m}$ : First, $T_{2 m}$ must be large enough, so that $\left(T_{0}, \ldots, T_{2 m}\right)$ dominates the given Hilbert seminorm. On the other hand, if $f_{v} \in \mathscr{S}_{v}$, $g_{\mu} \in \mathscr{S}_{\mu}, v+\mu=2 m$ and $v<m$, one must be able to estimate $T_{2 m}\left(f_{v}^{*} \times g_{\mu}\right)$ from above by $T_{2 v}\left(f_{v}^{*} \times f_{v}\right)^{1 / 2}$ times a continuous, invariant seminorm of $g_{\mu}$, vanishing on $\mathscr{L}_{S}^{(\mu)}$, 
in order to carry on with the construction. It is for this latter estimate that property $(R)$ is essential. A combination of Definition $4.1(1)$ with Proposition 3.7, and 4.1 (2) with Riesz' lemma implies the existence of a tempered, positive measure $d \varrho_{0}$ with support in $S$, and positive (unbounded) operators $A^{k}, k=1,2, \ldots$, on $L_{2}\left(S, d \varrho_{0}\right)$, such that

(a) $f \mapsto\|f\|_{k}:=\left(\int_{S} \overline{f(q)}\left(A^{k} f\right)(q) d \varrho_{0}(q)\right)^{1 / 2}$

and $f \mapsto\left(\int_{S}\left|A^{k} f(q)\right|^{2} d \varrho_{0}(q)\right)^{1 / 2}$ are continuous seminorms on $\mathscr{S}_{1}$.

(b) Every continuous seminorm on $\mathscr{S}_{1}$, which vanishes on $\mathscr{L}_{S}^{(1)}$, is dominated by $\|\cdot\|_{k}$ for some $k$.

Let $\varrho$ be another positive, tempered measure with support in $S$ and $k \in \mathbb{N}$. Let us call $(\cdot, \cdot)_{\varrho, k}^{(m)}$ the scalar product on $\mathscr{S}_{m}$ corrresponding to the Hilbert seminorm

$$
\|\cdot\|\left\|_{\varrho, k}^{(m)}:=\right\| \cdot\left\|_{2, \varrho}^{\sim} \otimes_{\sigma}\right\| \cdot\left\|_{k} \otimes_{\sigma} \ldots \otimes_{\sigma}\right\| \cdot \|_{k},
$$

where the tensor decomposition refers to the variables $q_{m}, \ldots, q_{1}$ defined in Sect. 2 . From Proposition 3.7 and Proposition 2.6 (iv), we know that every continuous, translationally invariant Hilbert seminorm on $\mathscr{S}_{m}$, vanishing on $\mathscr{L}_{S}^{(m)}$ can be dominated by a seminorm of this type. Define a distribution $R_{\varrho, k}^{(2 m)}$ in $\mathscr{S}_{2 m}^{\prime}$ by

$$
R_{\varrho, k}^{(2 m)}(f \times g)=\left(f^{*}, g\right)_{\varrho, k}^{(m)}
$$

for $f, g \in \mathscr{S}_{m}$ and extension by linearity and continuity to the whole of $\mathscr{S}_{2 m}$. By definition, $R_{\varrho, k}^{(2 m)}\left(f^{*} \times f\right)^{1 / 2}=\|f\|_{\varrho, k}^{(m)}$ for $f \in \mathscr{S}_{m}$, and we now want to estimate $R_{\varrho, k}^{(2 m)}\left(f_{v}^{*} \times g_{\mu}\right)$ from above for $f_{v} \in \mathscr{S}_{v}, g_{\mu} \in \mathscr{S}_{\mu}, v+\mu=2 m, v<m$. In order to do this, it is convenient to introduce different systems of coordinates on $\mathbb{R}^{\text {nd }}$. We had in Sect. 2 defined

$$
q_{k}=\sum_{i=n+1-k}^{n} p_{i}, \quad k=1, \ldots, n
$$

and we now define

$$
q_{k}^{\prime}=\sum_{i=1}^{k} p_{i}, \quad k=1, \ldots, n
$$

As independent variables in $\mathbb{R}^{n d}$, we may, for any $v, \mu$ with $v+\mu=n$, take $\left(q_{1}^{\prime}, \ldots, q_{v}^{\prime}, q_{\mu}, \ldots, q_{1}\right)$. A function $f$ of $\left(p_{1}, \ldots, p_{n}\right)$ can also be expressed in these coordinates; we indicate this by writing

$$
f\left(p_{1}, \ldots, p_{n}\right)=f_{v \mu}\left(q_{1}^{\prime}, \ldots, q_{v}^{\prime}, q_{\mu}, \ldots, q_{1}\right) .
$$

We note: If $f \in \mathscr{S}_{\nu}$ and $g \in \mathscr{S}_{\mu}$, then

$$
\left(f^{*} \times g\right)_{v \mu}\left(q_{1}^{\prime}, \ldots, q_{v}^{\prime}, q_{\mu}, \ldots, q_{1}\right)=\overline{f_{0 \nu}\left(-q_{v}^{\prime}, \ldots,-q_{1}^{\prime}\right)} g_{0 \mu}\left(q_{\mu}, \ldots, q_{1}\right) .
$$

Suppose now that $v+\mu=2 m, v<m$. The transformation of coordinates from $\left(q_{1}^{\prime}, \ldots, q_{v}^{\prime}, q_{\mu}, \ldots, q_{1}\right)$ to $\left(q_{1}^{\prime}, \ldots, q_{m}^{\prime}, q_{m}, \ldots, q_{1}\right)$ is given by

$$
q_{\lambda}^{\prime}=q_{\nu}^{\prime}+q_{\mu}-q_{2 m-\lambda}, \quad \lambda=v+1, \ldots, m,
$$


or conversely

$$
q_{\kappa}=q_{m}^{\prime}+q_{m}-q_{2 m-\kappa}^{\prime}, \quad \kappa=m+1, \ldots, \mu .
$$

Hence, we can also write

$$
\begin{aligned}
& \left(f^{*} \times g\right)_{m m}\left(q_{1}^{\prime}, \ldots, q_{m}^{\prime}, q_{m}, \ldots, q_{1}\right) \\
& \quad=\overline{f_{0 v}\left(-q_{v}^{\prime}, \ldots,-q_{1}^{\prime}\right)} g_{0 \mu}\left(q_{m}^{\prime}+q_{m}-q_{v}^{\prime}, \ldots, q_{m}^{\prime}+q_{m}-q_{m-1}^{\prime}, q_{m}, \ldots, q_{1}\right) .
\end{aligned}
$$

The distribution $R_{\varrho, k}^{(2 m)}$ can be written in the coordinates $\left(q_{1}^{\prime}, \ldots, q_{m}^{\prime}, q_{m}, \ldots, q_{1}\right)$ as

$$
\begin{aligned}
R_{\varrho, k}^{(2 m)}(f)= & \int \prod_{i=1}^{m} d q_{i}^{\prime} \prod_{j=1}^{m-1} d \varrho_{0}\left(q_{j}\right) d \varrho\left(q_{m}\right) \prod_{i=1}^{m} \delta\left(q_{i}^{\prime}+q_{i}\right) \\
& \cdot \prod_{j=1}^{m-1} A_{j}^{k} f_{m m}\left(q_{1}^{\prime}, \ldots, q_{m}^{\prime}, q_{m}, \ldots, q_{1}\right) .
\end{aligned}
$$

Here $\prod_{j=1}^{m-1} A_{j}^{k}$ stands for the tensor product of the $A^{k}$, operating on $f_{m m}$ as a function of the last $(m-1)$ variables, with the other variables fixed. Thus, if $f \in \mathscr{S}_{v}$ and $g \in \mathscr{S}_{\mu}, v+\mu=2 m$ and $\nu<m$, we have

$$
\begin{aligned}
\left|R_{\varrho, k}^{(2 m)}\left(f^{*} \times g\right)\right|=\mid & \mid \prod_{j=1}^{m-1} d \varrho_{0}\left(q_{j}\right) d \varrho\left(q_{m}\right) \overline{f_{0 v}\left(q_{v}, \ldots, q_{1}\right)} \\
& \cdot \prod_{j=1}^{m-1} A_{j}^{k} g_{0 \mu}\left(q_{v}, \ldots, q_{m-1}, q_{m}, \ldots, q_{1}\right) \mid \\
\leqq & \left(\int \prod_{j=1}^{v} d \varrho_{0}\left(q_{j}\right)\left|f_{0 v}\left(q_{v}, \ldots, q_{1}\right)\right|^{2}\right)^{1 / 2} \\
& \cdot\left(\int \prod_{j=1}^{v} d \varrho_{0}\left(q_{j}\right) \mid \int \prod_{i=v+1}^{m-1} d \varrho_{0}\left(q_{i}\right) d \varrho\left(q_{m}\right)\right. \\
& \left.\left.\cdot \prod_{l=1}^{m-1} A_{j}^{k} g_{0 \mu}\left(q_{v}, \ldots, q_{m-1}, q_{m}, \ldots, q_{1}\right)\right|^{2}\right)^{1 / 2} .
\end{aligned}
$$

The first factor is $\leqq R_{\varrho_{0}, 0}^{(2 v)}\left(f^{*} \times f\right)^{1 / 2}$. The second factor is a $\tilde{\mathfrak{I}}_{2, s^{-c o n t i n u o u s}}$ seminorm of $g$ : Suppose $g\left(q_{\mu}, \ldots, q_{1}\right)=\prod_{j=1}^{\mu} g_{j}\left(q_{j}\right)$. The second factor is then

$$
\begin{gathered}
\prod_{j=1}^{v-1}\left(\int\left|A^{k} g_{j}\left(q_{j}\right)\right|^{2} d \varrho_{0}\left(q_{j}\right)\right)^{1 / 2}\left(\int d \varrho_{0}\left(q_{v}\right)\left|g_{\mu}\left(q_{v}\right) A^{k} g_{v}\left(q_{v}\right)\right|^{2}\right)^{1 / 2} \\
\cdot \prod_{i=v+1}^{m-1}\left|\int d \varrho_{0}\left(q_{i}\right) g_{2 m-i}\left(q_{i}\right) A^{k} g_{i}\left(q_{i}\right)\right|\left|\int d \varrho(q) g_{m}(q)\right| \\
\leqq \\
\prod_{j=1}^{m-1}\left(\int\left|A^{k} g_{j}\left(q_{j}\right)\right|^{2} d \varrho_{0}\left(q_{j}\right)\right)^{1 / 2} \int d \varrho(q)\left|g_{m}(q)\right| \\
\cdot \prod_{i=m+1}^{\mu-1}\left(\int d \varrho_{0}\left(q_{i}\right)\left|g_{i}\left(q_{i}\right)\right|^{2}\right)^{1 / 2} \sup _{q \in S}\left|g_{\mu}(q)\right|
\end{gathered}
$$


The second factor can therefore be estimated by a $\pi$-tensor product of continuous seminorms on $\mathscr{S}_{1} / \mathscr{L}_{s}^{(1)}$. Moreover, it is an invariant Hilbert seminorm, so Proposition 3.7 implies that it is $\tilde{\mathfrak{I}}_{2, \mathrm{~s}}$-continuous. With these estimates the functional $T$ can be constructed in exactly the same way as in the proof of Theorem 1 in [7]. We define $T_{2 m+1}=0$ and $T_{2 m}=\lambda_{m} R_{\varrho_{m}, k_{m}}^{(2 m)}$, where the constants $\lambda_{m}$ and $k_{m}$ and the measures $\varrho_{m}$ are determined by induction over $m$, such that $T$ is positive and dominates the given $\tilde{\mathfrak{T}}_{2, s^{-}}$-continuous seminorm $\|\cdot\|$.

Finally, we show that condition $(R)$ is necessary for the first part of the theorem. This is based on the simple observation, that if $T$ is an invariant, positive functional in $\mathscr{L}_{S}^{\perp}$, then for every fixed $h \in \mathscr{S}_{1}$ the sequence of scalar products

$$
\begin{aligned}
& (f, g)_{0}=T_{2}\left(f^{*} \times g\right) \\
& (f, g)_{k}=T_{2^{k}+2}\left(f^{*} \times h^{*} \times \ldots h \times g\right)
\end{aligned}
$$

has properties (1) and (2) of Definition 4.1 (with $k^{\prime}=k+1$ ). Now for any basis of Hilbert seminorms $\|\cdot\|_{k}$ on $\mathscr{S}_{1} / \mathscr{L}_{S}^{(1)}$, we may construct a Hilbert seminorm $\|\cdot\|$ on $\mathscr{L} / \mathscr{L}_{S}$ such that $\left\|h^{*} \times \ldots h \times g\right\|=\|g\|_{k}$, where $h$ is some fixed function in $\mathscr{P}_{1}$ occurring $2^{k-1}$ times in the product. If this seminorm can be dominated by an invariant, positive functional $T$ in $\mathscr{L}_{S}^{\perp}$, then the scalar products defined above form a basis.

4.6. Remark. No attempt has been made to obtain an optimal estimate for $T$ from above. The structure of the $n$-point distributions $T_{n}$ used in the construction is particularly simple, but much smaller functionals (which nevertheless dominate a given seminorm) can be defined at the cost of introducing more complicated expressions, cf. Satz 4.9 in [25]. A somewhat different method for constructing positive functionals is used in [13]. It proceeds also by induction, but care is taken to make the piece added at each step as small as possible. It seems, however, that the incorporation of translation invariance and spectrum condition would also make this construction fairly complicated.

\section{The Linear Span of the Invariant States}

We can now give a characterization of those linear functionals in $\mathscr{S}^{\prime}$, which can be split into translationally invariant, positive linear functionals fulfilling a spectrum condition.

5.1. Theorem. Suppose $S \subset \mathbb{R}^{d}$ satisfies condition $(R)$, and let $T$ be a translationally invariant linear functional on $\mathscr{S}$. The following are equivalent:

(i) There are positive, translationally invariant functionals $T_{j} \in \mathscr{L}_{S}^{\perp}, j=1, \ldots, 4$, such that $T=\left(T_{1}-T_{2}\right)+i\left(T_{3}-T_{4}\right)$.

(ii) There is a translationally invariant, continuous Hilbert seminorm $\|\cdot\|$ on $\mathscr{S}$, vanishing on $\mathscr{L}_{S}$, such that $\left|T\left(\underline{f}^{*} \times \underline{g}\right)\right| \leqq\|f\|\|g\|$ for all $\underline{f}, g \in \underline{\mathscr{Q}}$.

(iii) There is a continuous seminorm $\|\cdot\|$ on $\mathscr{Q}$ such that

$$
\left|\int T\left(f \times \alpha_{a} \underline{g}\right) h(a) d a\right| \leqq\|\underline{f}\|\|g\| \sup _{q \in S}|\tilde{h}(q)|
$$

for all $\underline{f}, g \in \underline{\mathscr{S}}, h \in \mathscr{S}_{1}$. 
Proof. The implication (i) $\Rightarrow$ (ii) follows from the Cauchy-Schwarz inequality for the positive functionals $T_{j}$. The equivalence of (ii) and (iii) follows from Propositions 3.6 and 3.7. Note that (iii) implies that $T \in \mathscr{L}_{S}^{\perp}$, so we may assume that the seminorm $\|\cdot\|$ vanishes on $\mathscr{L}_{S}$. Suppose $T$ satisfies (ii). The same holds for its hermitian and antihermitian part, so we can assume $T \in \mathscr{S}_{h}^{\prime}$. By Theorem 4.5 there is a positive, translationally invariant functional $\hat{T} \in \mathscr{L}_{S}^{\perp}$ such that $\left|T\left(\underline{f}^{*} \times \underline{f}\right)\right| \leqq \hat{T}\left(\underline{f}^{*} \times \underline{f}\right)$ for all $\underline{f} \in \mathscr{\mathscr { L }}$. Then $T=(T+\hat{T})-\hat{T}$ is the desired splitting of $T$, so (ii) $\Rightarrow(\mathrm{i})$.

5.2. Remarks. (1) Condition (iii) is easy to check, so this theorem leads to a much larger class of invariant states than those constructed explicitly in the proof of Theorem 4.5. (2) Besides implying that the function $a \mapsto T\left(f \times \alpha_{a} g\right)$ is the Fourier transform of a bounded measure for fixed $f, g$, condition (iii) says also that all these measures are uniformly bounded for $\|\bar{f}\|,\|g\| \leqq 1$. Examples can be given of functionals $T \in \mathscr{S}_{h}^{\prime}$ with the following properties: a) $T$ is translationally invariant. b) There exists a translationally invariant, continuous seminorm $\|\cdot\|$ on $\mathscr{S}$ such that $|T(\underline{f} \times \underline{g})| \leqq\|\underline{f}\|,\|\underline{g}\|$ for all $\underline{f}, \underline{g}$. (In particular, $T$ is the difference of two positive functionals [7].) c) For all $f, \underline{g}$ the function $a \mapsto T\left(\underline{f} \times \alpha_{a} g\right)$ is the Fourier transform of a bounded measure. d) $T$ is not of the form $T_{1}-T_{2}$ with translationally invariant, positive functionals $T_{j}$.

5.3. Theorem. Suppose $S$ satisfies condition $(R)$.

(i) $\hat{\mathscr{M}}_{S}$ is the intersection of all $\operatorname{Ker} T$ with $T \in \hat{\mathscr{M}}_{S}^{\perp} \cap \mathscr{S}^{+{ }^{\prime}}$. In other words: The translationally invariant states in $\mathscr{L}_{S}^{\perp}$ span a dense subspace of $\hat{M}_{S}^{\perp}=\mathscr{L}_{S}^{\perp} \cap \mathscr{P}_{\text {inv }}^{\prime}$.

(ii) $\mathscr{L}_{S}$ is the intersection of the left kernels $\left\{\underline{f} \mid T\left(\underline{f}^{*} \times \underline{f}\right)=0\right\}$ of translationally invariant states $T$ in $\mathscr{L}_{S}^{\perp}$.

Proof. (i) The two forms of the assertion are equivalent by the bipolar theorem. We show that $\hat{\mathscr{M}}_{S}=\cap\{\operatorname{Ker} T\}$. The inclusion $C$ is clear. Consider now a functional $T$ with $T_{n}=0$ for $n \neq N>0$, and $\tilde{T}_{N}\left(p_{1}, \ldots, p_{N}\right)=\delta\left(p_{1}+\ldots+p_{N}\right)$ $\prod_{k=1}^{N} \delta\left(\bar{q}_{k}-\left(p_{N+1-k}+\ldots+p_{N}\right)\right)$, where the $\bar{q}_{k}$ are some fixed points in $S$. By Proposition 2.6 (ii), $\hat{\mathscr{M}}_{S}$ is the intersection of the kernels of all such functionals (and $T=(1,0, \ldots))$. But such functionals obviously satisfy 5.1 (iii) and are thus decomposable into functionals in $\mathscr{L}_{S}^{\perp} \cap \mathscr{L}^{+\prime}$. (ii) Obviously, there exist continuous, invariant Hilbert seminorms on $\mathscr{L}$, which vanish exactly on $\mathscr{L}_{S}$ (cf. e.g. the proof of Theorem 4.5). If $T$ dominates such a seminorm, then $\mathscr{L}_{S}$ is equal to the left kernel of $T$.

5.4. Remark. Although condition $(R)$ is necessary for Theorem 4.5, the last theorem is a much weaker statement and is in fact true for quite arbitrary $S$. One way to prove this is to modify Theorem 4.5 by considering only a subclass of invariant Hilbert seminorms and construct the dominating positive functionals in a similar way as in Satz 4.9 and 4.10 in [25]. Another method for constructing invariant functionals is discussed in [16], where an analog of Theorem 5.3 for Poincaré invariant states with spectrum condition is proven. 


\section{On Totally Symmetric Functionals and Euclidean Invariant Measures}

In Euclidean field theory one is interested in linear functionals on $\mathscr{S}$ which are totally symmetric under permutations of the arguments (equivalently: functionals on the totally symmetric tensor algebra over $\mathscr{S}$ ). In this formalism, the following question arises [26-28]: when has such a functional $T$ a representation by a positive, or more generally, complex measure $d \mu$ on the space of (real) tempered distributions $\mathscr{S}_{R}^{\prime}$, in the sense that

$$
T_{n}\left(f_{1} \times \ldots \times f_{n}\right)=\int_{\mathscr{S}_{R}^{\prime}} \omega\left(f_{1}\right) \ldots \omega\left(f_{n}\right) d \mu(\omega)
$$

for all $f_{1}, \ldots, f_{n} \in \mathscr{S}_{1}, n \in \mathbb{N}$ ? A further question is: when is there a representing measure which is invariant under the natural action of the Euclidean group on $\mathscr{S}_{R}^{\prime}$ ? The first problem is well understood [26-28]. If invariance is required, however, only partial answers are known. We now want to make a series of remarks on this problem.

The group of Euclidean transformations of $\mathbb{R}^{d}$ operates in a natural way as a group of automorphisms of $\mathscr{\mathscr { S }}$, and we call a functional on $\mathscr{\mathscr { S }}$ Euclidean invariant if it is invariant under this action. Recall furthermore that a totally symmetric linear functional on $\mathscr{S}$ is called strongly positive, if it is positive on all positive polynomials in the symmetric tensor algebra. [This is somewhat stronger than simple positivity, i.e. $T\left(\underline{f}^{*} \times \underline{f}\right) \geqq 0$ for all $\underline{f}$.]

6.1. Proposition. A totally symmetric functional $T \in \mathscr{S}^{\prime}$ has a representation by a positive, Euclidean invariant measure, if and only if $T$ is strongly positive and Euclidean invariant on $\mathscr{L}$.

Proof. $T$ has a representation by a positive measure if and only if $T$ is strongly positive [26-28]. This measure is not necessarily unique. However, it follows from positivity that the collection of all such measures for a given $T$ is a bounded (even compact) set in the weak topology defined by polynomially bounded cylinder functions on $\mathscr{S}_{R}^{\prime}$. One thus obtains an invariant measure by averaging over the Euclidean group, which is amenable. See [26], Theorem 3.8 for details.

6.2. Remark. The Schwinger functions of Euclidean quantum field theory do not from the outset define a linear functional on $\mathscr{S}$, but rather on a certain subspace $\mathscr{S}_{0}$, cf. e.g. $[17,26]$. Now the following situation can in principle occur: An invariant linear functional on $\mathscr{S}_{0}$ has an extension to a strongly positive functional on $\mathscr{I}$, but no extension which is both invariant and strongly positive. This is somewhat puzzling at first sight, since one might think that an averaging procedure as in the last proposition would lead to an invariant extension. However, in general $T\left(\alpha_{a} f\right)$ will not be a bounded function of the translations $a$ for $\underline{f} \in \mathscr{\mathscr { S }}$, even if $T$ is translationally invariant on $\mathscr{S}_{0}$. Positivity of $T$ is of no help in establishing boundedness unless every $f \in \mathscr{L}$ can be dominated by a positive element in $\mathscr{S}_{0}$. For the space $\mathscr{S}_{0}$ of interest in Euclidean field theory (functions vanishing at coinciding arguments) this is not the case.

We now come to more general representations by Euclidean invariant complex measures. In [17] it was shown that a totally symmetric functional $T \in \mathscr{L}^{\prime}$ has a 
representation by a complex measure if and only if there are continuous seminorms $\|\cdot\|_{k}, k=1,2, \ldots$, on $\mathscr{S}_{1}$ such that

$$
\left|T_{n}\left(f_{1} \times \ldots \times f_{n}\right)\right| \leqq\left\|f_{1}\right\|_{1} \ldots\left\|f_{n}\right\|_{n}
$$

for all $f_{1}, \ldots, f_{n} \in \mathscr{S}_{1}, n \in \mathbb{N}$. Now suppose $T$ is Euclidean invariant on $\mathscr{\mathscr { S }}$. One has in any case a representation by a rotationally invariant measure, since the rotation group is compact and one can integrate over it. For the translation group the situation is more involved:

6.3. Proposition. In order that a totally symmetric translationally invariant functional $T \in \mathscr{S}^{\prime}$ has a representation by a translationally invariant complex measure, it is necessary that there exist seminorms $\|\cdot\|_{k}$ on $\mathscr{S}_{1}$ such that $\|\cdot\|_{1}$ is $\tilde{\mathfrak{I}}_{2}$-continuous, $\|\cdot\|_{k}$ is $\mathfrak{I}_{\mathrm{inv}}$-continuous for $k=2,3, \ldots$, and

$$
\left|T_{n}\left(f_{1} \times \ldots \times f_{n}\right)\right| \leqq\left\|f_{1}\right\|_{1} \ldots\left\|f_{n}\right\|_{n}
$$

for all $f_{1}, \ldots, f_{n} \in \mathscr{S}_{1}, n \in \mathbb{N}$.

Proof. By [30], Lemma 3.3, one can write every invariant measure as a linear combination of positive invariant measures. Hence one needs only check the condition for such measures $d \mu$. But in that case we can take

$$
\|f\|_{k}=\left(\int_{\mathscr{S}_{R}^{\prime}}|\omega(f)|^{2^{k}} d \mu(\omega)\right)^{1 / 2^{k}} .
$$

6.4. An Example. Consider the time-ordered functions of a free field of mass $m$. It is obvious that an estimate of the type $\left({ }^{*}\right)$ is fulfilled, because the $n$-point function is a finite sum of products of 2-point functions. Hence there is a representation by a complex measure. On the other hand there is no representation by a translationally invariant measure, because the Fourier transform of the 2-point function, $T_{2}(p)=\lim _{\varepsilon \rightarrow 0^{+}}\left(p^{2}-m^{2}+i \varepsilon\right)^{-1}$, is not a measure in $p$ (and therefore not continuous in $\left.\tilde{\mathfrak{T}}_{2}\right)$. The same argument applies if the field is not free, as long as there is a discrete mass appearing in the 2-point function.

This example shows that the existence of an invariant mean on a group is not necessarily of any use for the construction of invariant functionals. What happens in the example above is that the translates of a representing measure do not form a bounded set.

It is not known if the condition in Proposition 6.3 is also sufficient. In any case, it follows from Theorem 5.1 that $T$ can be split into invariant, positive functionals on $\mathscr{L}$. But these functionals need not be symmetric nor strongly positive. In [16] it is shown that the functionals which have a representation by an invariant measure are at least dense in the space of all invariant totally symmetric functionals.

\section{Appendix}

In this appendix we prove Proposition 2.6.

Lemma. Let $A \subset \mathbb{R}^{n}$ and $B \subset \mathbb{R}^{m}$ be closed sets. Define $\mathscr{N}_{A}=\left\{f \in \mathscr{S}\left(\mathbb{R}^{n}\right) \mid f=0\right.$ on $\left.A\right\}$ and in the same way $\mathscr{N}_{B} \subset \mathscr{S}\left(\mathbb{R}^{m}\right)$ and $\mathscr{N}_{A \times B} \subset \mathscr{S}\left(\mathbb{R}^{n+m}\right)$. Put $\mathscr{N}_{A, B}=$ closure of 
$\left(\mathscr{N}_{A} \otimes \mathscr{S}\left(\mathbb{R}^{m}\right)+\mathscr{S}\left(\mathbb{R}^{n}\right) \otimes \mathscr{N}_{B}\right)$. Then
(a) $\mathscr{N}_{A, B}^{\perp} \simeq \mathscr{N}_{A}^{\perp} \hat{\otimes} \mathscr{N}_{B}^{\perp}$,
(b) $\mathscr{N}_{A, B} \simeq \mathscr{N}_{A \times B}$,
(c) $\left.\mathscr{S}\left(\mathbb{R}^{n+m}\right) / \mathscr{N}_{A \times B} \simeq \mathscr{S}\left(\mathbb{R}^{n}\right) / \mathscr{N}_{A}\right) \hat{\otimes}\left(\mathscr{S}\left(\mathbb{R}^{m}\right) / \mathscr{N}_{B}\right)$.

Here $\simeq$ stands for a natural isomorphism between topological vector spaces.

Proof. By [29], p. 236, we have $\mathscr{N}_{A, B}^{\perp} \simeq\left(\mathscr{N}_{A} \hat{\otimes} \mathscr{S}\left(\mathbb{R}^{m}\right)\right)^{\perp} \cap\left(\mathscr{S}\left(\mathbb{R}^{n}\right) \hat{\otimes} \mathscr{N}_{B}\right)^{\perp}$. By [20], 7.4.3, we can write every $T \in \mathscr{S}\left(\mathbb{R}^{n+m}\right)^{\prime}=\left(\mathscr{S}\left(\mathbb{R}^{n}\right) \hat{\otimes} \mathscr{S}\left(\mathbb{R}^{m}\right)\right)^{\prime}$ as $T=\sum_{i=1}^{\infty} t_{i} \otimes s_{i}$ with $t_{i} \in \mathscr{S}\left(\mathbb{R}^{n}\right)^{\prime}, s_{i} \in \mathscr{S}\left(\mathbb{R}^{m}\right)^{\prime}$. Moreover, we can take the $t_{i}$ linearily independent, so if $T \in \mathscr{N}_{A, B}^{\perp}$, then $s_{i} \in \mathscr{N}_{B}^{\perp}$ for all $i$. Writing $T=\sum_{i=1}^{\infty} t_{i}^{\prime} \otimes s_{i}^{\prime}$ with $s_{i}^{\prime}$ linearily independent in $\mathscr{N}_{B}^{\perp}$, we get $t_{i}^{\prime} \in \mathscr{N}_{A}^{\perp}$ and thus $T \in \mathscr{N}_{A}^{\perp} \hat{\otimes} \mathcal{N}_{B}^{\perp}$, showing (a). (b) From the bipolar theorem and the fact that $\mathscr{S}\left(\mathbb{R}^{n}\right) \hat{\otimes} \mathscr{S}\left(\mathbb{R}^{m}\right) \simeq \mathscr{S}\left(\mathbb{R}^{n+m}\right)$, it suffices to compare $\mathscr{N}_{A, B}^{\perp}$ and $\mathscr{N}_{A \times B}^{\perp}$. It is clear that $\mathscr{N}_{A \times B}^{\perp} \subset \mathscr{N}_{A, B}^{\perp}$, and from (a) we know that $T \in \mathscr{N}_{A, B}^{\perp}$ can be written $T=\sum t_{i} \otimes s_{i}$ with $t_{i} \in \mathscr{N}_{A}^{\perp}, s_{i} \in \mathscr{N}_{B}^{\perp}$. But such $T$ are obviously in $\mathscr{N}_{A \times B}^{\perp}$. (c) Since both sides are nuclear Fréchet spaces and thus reflexive, it suffices to compare the duals, so (c) follows from (a) by [29], p. 254.

Proof of Proposition 2.6. From the definition of $\mathscr{L}_{S}$ it is clear that $\mathscr{L}_{S}=\left\{\underline{f} \mid f_{0}=0\right.$, $f_{n} \in$ closure of $\left.\sum_{v+\mu=n-1} \mathscr{S}^{\hat{\otimes} v} \otimes \mathscr{N}_{S} \otimes \mathscr{S}^{\hat{\otimes} \mu}\right\}$. This shows (i) by part (b) of the lemma.

In the same way one gets (ii), using Proposition 2.1 (iv). From this it is evident that $\hat{\mathscr{M}}_{S}$ is *-invariant, so $\mathscr{L}_{S}^{*}$ and thus $\mathscr{M}_{S} \subset \hat{\mathscr{M}}_{S}$. Suppose $S \cap-S=\{0\}$ and 0 is an isolated point of $S$. Assume for a moment that $S$ is compact. We can then find a function $\chi \in \mathscr{S}$ of compact support such that $\chi=0$ on $S$ and $\chi=1$ on $(-S) \backslash\{0\}$. If $f_{n}$ vanishes on $\{0\} \times S \times \ldots \times S$, we can write $f_{n}\left(q_{n}, \ldots, q_{1}\right)=\chi\left(q_{n}\right) f_{n}\left(q_{n}, \ldots, q_{1}\right)$ $+\left(1-\chi\left(q_{n}\right)\right) f_{n}\left(q_{n}, \ldots, q_{1}\right)$. The first term is in $\mathscr{L}_{S}$ and the second one in $\mathscr{L}_{S}^{*}$. For non-compact $S$ we apply this to functions in $\mathscr{M}_{S}$ of compact support. Since these are dense, we have in any case $\hat{\mathscr{M}}_{S}=\operatorname{closure}\left(\mathscr{L}_{S}+\mathscr{L}_{S}^{*}\right)=\mathscr{M}_{S}$. Part (iv) follows from part (c) of the lemma. It should be noted, that the tensor decomposition is with respect to the variables $q_{k}$.

Acknowledgements. This paper is an extended version of a part of the Habilitationsschrift of the author, Univ. of Göttingen 1978 [25]. I would like to thank Professor H. J. Borchers and the other members of the Institute for Theoretical Physics in Göttingen, and also R. Magnus, J. Arason and R. Axelsson of the Science Institute in Reykjavik for helpful discussions.

\section{References}

1. Borchers, H.J. : Nuovo Cimento 24, 214-236 (1962)

2. Uhlmann, A.: Wiss. Zeitschr. Karl-Marx-Univ. 11, 213-217 (1962)

3. Borchers, H.J.: Algebraic aspects of Wightman field theory. In: Statistical mechanics and field theory, Sen, R., Weil, C. (eds.). New York: Halsted Press 1972

4. Lassner, G., Uhlmann, A.: Commun. Math. Phys. 7, 152-159 (1968)

5. Lassner, G.: On the structure of the test function algebra. JINR preprint, Dubna E 2-5254 (1970)

6. Wyss, W.: Commun. Math. Phys. 27, 223-234 (1972)

7. Yngvason, J.: Commun. Math. Phys. 34, 315-333 (1973) 
8. Hoffmann, G.: Die Testfunktionenalgebra $\mathscr{S}_{\otimes}$ und ihre Anwendungen in der axiomatischen Quantenfeldtheorie. Dissertation, Univ. Leipzig, 1975

9. Dubois-Violette, M. : Commun. Math. Phys. 43, 225-254 (1975)

10. Dubois-Violette, M.: Commun. Math. Phys. 54, 151-172 (1977)

11. Brüning, E.: Commun. Math. Phys. 58, 139-194 (1978)

12. Brüning, E.: The $N$-field irreducible part of an $n$-point functional. Ann. Inst. Henri Poincare 34, 309-328 (1981)

13. Brüning, E.: On the construction of fields and the topological role of Jacobi fields. Preprint, CERN-TH 2830, 1980

14. Alcántara, J., Dubin, D.: I*-Algebras and their representation. Publ. RIMS, Kyoto Univ. (to appear)

15. Lassner, G.: Algebras of unbounded operators and representations. In: Proceedings of the international conference on operator algebras, ideals and their applications in theoretical physics, Leipzig, 1977. Baumgärtel, H. et al. (eds.). Leipzig: Teubner 1978

16. Yngvason, J.: A method for constructing invariant states on Borchers' tensor algebra (in preparation)

17. Borchers, H.J., Yngvason, J.: Commun. Math. Phys. 47, 197-213 (1976)

18. Schwartz, L.: Théorie des distributions. Paris: Hermann 1966

19. Trèves, F.: Topological vector spaces, distributions and kernels. New York-London: Academic Press 1967

20. Pietsch, A.: Nuclear locally convex spaces. Berlin, Heidelberg, New York: Springer 1972

21. Vogt, D.: Subspaces and quotient spaces of $(s)$. In: Functional analysis, surveys and recent results. Bierstedt, K.D., Fuchssteiner, B. (eds.). Amsterdam: North-Holland 1977

22. Tougeron, J.C.: Ideaux de fonctions differentiables. Berlin, Heidelberg, New York: Springer 1977

23. Zerner, M.: C.R. Acad. Sci. Paris 268, A 218-220 (1969)

24. Baouendi, M.S., Goulaouic, C.: Ann. l'Inst. Fourier 21, 149-173 (1971)

25. Yngvason, J.: Über den algebraischen Formalismus in der Wightmanschen Quantenfeldtheorie. Habilitationsschrift, Univ. of Göttingen, 1978

26. Challifour, J., Slinker, S. : Commun. Math. Phys. 43, 41-58 (1975)

27. Borchers, H.J., Yngvason, J.: Commun. Math. Phys. 43, 255-291 (1975)

28. Hegerfeldt, G.C.: Commun. Math. Phys. 45, 133-135 (1975)

29. Koethe, G.: Topological vector spaces I. Berlin, Heidelberg, New York: Springer 1969

30. Yngvason, J.: Rep. Math. Phys. 13, 101-115 (1978)

Communicated by $\mathrm{H}$. Araki

Received January 14, 1981 